\title{
Engineering of MOFs and MOF-derived Active Materials for Energy and Water Technologies
}

\author{
John Wang \\ Department of Materials Science and Engineering \\ National University of Singapore
}

Energy and water technologies are strongly related in terms of requirement for the types of advanced materials. Water and wastewater treatment processes are among the most energy intensive process. We have been exploring a class of 2D materials for both energy and water technologies, by engineering of MOFs (Metal Organic Frameworks) and MOF-derived Materials. For the next generation energy technologies, such as electrical vehicles, green energy and energy storage, these 2D materials can be made into 2D layered, 2D non-layered and 2D holey morphologies, and their compositions can be tuned into carbon-based, metal sulfides, phosphides, nitrides and carbonitrides. For high performing energy storage and conversion, both the number and population of active sites can be maximized by the approaches such as site-engineering, facet manipulation, and control in pore size, pore size distribution and surface area. Indeed, MOFs can be grown into various 3D, 2D, 1D and 0D morphologies on conducting and ceramic substrates, where the great flexibility in control over varying length scales from atomic scale up to bulk structure allows access to an almost endless variety of MOF-based and MOFderived active materials. MOF-derived 2D active materials are shown to be exceptionally high performing as electrodes in supercapacitors and rechargeable batteries. Another potentially important application for these 2D active materials is in electrocatalysis, where they have demonstrated outstanding behavior, which enable them to function as the efficient cathodes of metal- air batteries, such as Li-air and $\mathrm{Zn}$-air batteries. 2D layered, 2D non-layered and 2D holey active materials can be fabricated into either free-standing forms, nano- or micro-powders, grown on appropriate conducting substrates, or assembled together with other active materials. In parallel, MOFs and MOF-derived materials are shown among the efficient active top layers for flux and anti-fouling in the multilayer ceramic membrane structures. In this talk, I present the latest development of these $2 \mathrm{D}$ active materials conducted in our lab and the key understandings made. 